\title{
Enhanced light backscattering in thermally poled plasmonic nanocomposites and its application to vapour sensing
}

\author{
Olivier Deparis $^{1 *}$, Martynas Beresna ${ }^{2}$, Costantino Corbari ${ }^{2}$ and Peter G. Kazansky ${ }^{2}$ \\ ${ }^{1}$ Research Centre in Physics of Matter and Radiation (PMR), Univ. of Namur (FUNDP), B-5000 Namur, Belgium \\ 2 Optoelectronics Research Centre, University of Southampton, SO17 1BJ, United Kingdom \\ *olivier.deparis@fundp.ac.be
}

\begin{abstract}
We show theoretically that gold nanoparticles embedded in glass can exhibit enhanced light backscattering in presence of leaky waveguide and report on selective vapour sensing in thermally poled nanocomposite in which this concept is implemented.

(C2010 Optical Society of America

OCIS codes: (290.1350) Backscattering; (160.4236) Nano-materials; (160.1245) Artificially engineered materials
\end{abstract}

\section{Introduction}

Recently, enhanced interaction of light with gold nanoparticles embedded in glass was observed in thermally poled nanocomposite glass and exploited to demonstrate a novel plasmonic saturable absorber device with state-ofthe art performances [1]. The enhanced interaction was due to the presence of a leaky waveguide which was created during poling and acted as a monolithic analogue to the double-layer Kretschmann geometry used to excite surface plasmons in a metallic film. In ref. [1], the coupling of light into the leaky waveguide mode was shown to lead to nonlinear surface plasmon resonant absorption in gold nanoparticles but it was also pointed out that this coupling was associated with the onset of strong light scattering (1\% of incident power). Actually, enhanced scattering was observed in the backward direction as well with interesting sensing characteristics [2]. We report here on selective vapour sensing of a nanocomposite sample which contained a random arrangement of spheroid nanoparticles. In order to prove the existence of backscattering in poled plasmonic nanocomposite, we performed a theoretical study of the light scattering from a glass film containing gold nanoparticles in presence of leaky waveguide. For a conceptual proof, we considered three dimensional (3D) periodic arrangements of spherical nanoparticles and we calculated collective scattering properties using electromagnetic computational method.

\section{Sample characteristics and theoretical model}

The sample used in the experiments contained randomly dispersed spheroid gold nanoparticles ( $\sim 15 \mathrm{~nm}$ diameter, $\sim 2.3 \%$ volume filling fraction) in a sol-gel silica film ( $\sim 130 \mathrm{~nm}$ thickness) on top of a thick soda-lime glass substrate. The theoretical model consisted of a $\mathrm{SiO}_{2}$ film (refractive index $n_{\text {host }}=1.440$ ) in which spherical gold nanoparticles (diameter $b=15 \mathrm{~nm}$ ) were arranged periodically (Fig. 1). The 3D periodic arrangement of particles was built by stacking $N=8$ layers of 2D square array of particles (thickness $b$, spatial period $a$ ) with alternate shifts of half a period in both lateral directions from one layer to the next (such an array was thought to be not too far from actual particle arrangements, except for randomness in particle positions and clustering of some particles). Two very thin $\mathrm{SiO}_{2}$ layers (thickness $h=5 \mathrm{~nm}$ ) were added on top and bottom in order to obtain a total thickness $(d=N \times b+2 \times h)$ equal to the actual film thickness. The gold volume filling fraction was given by $f=4 / 3 \pi \times b^{3} /\left(a^{2} \times d\right)$. The film laid on top of a glass substrate $\left(n_{\text {sub }}=1.488\right)$, which was considered as the semi-infinite incidence medium (in experiments, light was launched from the sample side into the substrate [1,2]). Between the film and the substrate, a buried leaky waveguide layer was inserted (thickness $d_{\text {depl }}=2 \mu \mathrm{m}$ ) which represented the ion-depleted layer created during poling. The refractive index in this layer was slightly lower ( $\delta \sim 1 \%)$ than in the unmodified substrate regions, resulting in leaky waveguide. The plane of incidence was chosen as the $(x, z)$ plane, the incidence angle was noted $\theta$ (angle inside the substrate) and the incident light polarization was assumed to be transverse electric (TE). The wavelength of the light was taken equal to $\lambda=532 \mathrm{~nm}$, as in experiments. At this wavelength, the gold refractive index was equal to $n_{A u}=0.467+\mathrm{i} \times 2.407$. The $3 \mathrm{D}$ transfer-matrix (TM) method was used to solve exactly the problem of electromagnetic wave propagation by performing a
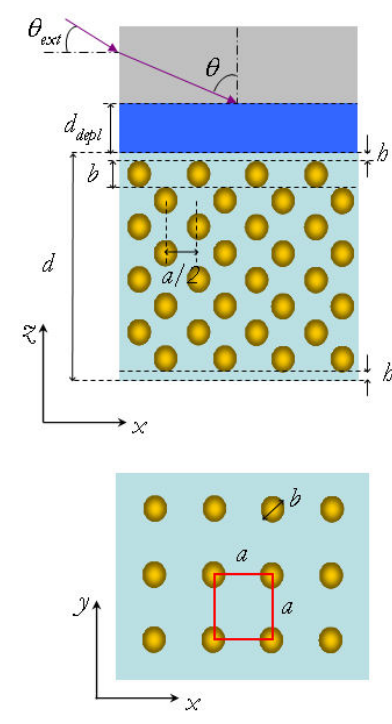

Fig. 1 Model of thermally poled nanocomposite sample: soda lime glass substrate (grey), leaky waveguide layer (dark blue), gold nanoparticles (yellow) embedded in sol-gel silica matrix (light blue). 
plane wave expansion of the dielectric function in both lateral $(x, y)$ directions. In the case of metal clusters, the non vanishing imaginary part of the refractive index required to use a much larger number of plane waves than in the case of dielectrics. In this work, we used up to $16 \times 16$ plane waves (diffraction directions) to achieve convergence within reasonable computation times.

\section{Results}

In the case of backscattering and for a square array of point scatterers, the momentum conservation law imposed: $\boldsymbol{k}_{g}=2 \times \boldsymbol{k}_{\text {inc } \|}\left(\boldsymbol{k}_{g}\right.$ : reciprocal lattice wavevector, $\boldsymbol{k}_{\text {inc } \|}$ : in-plane component of incident wavevector). For an incident light in the $(x, z)$ plane, the resulting phase matching condition allowed us to estimate the shortest period that was required for backscattering: $a=\lambda /\left(2 \times n_{\text {sub }} \times \sin \theta\right)$. At grazing incidence $(\theta=\pi / 2)$, for which enhanced backscattering was observed experimentally [2], the period was estimated to be $a \sim 179 \mathrm{~nm}$. Transfer-matrix numerical simulations (Fig. 2) were performed in order to validate this prediction and to quantify the backscattering efficiency. Leaky waveguide resonances were observed at grazing incidence in the angular spectra of the reflectance in the specular direction, for a wide range of periods $(a=180-215 \mathrm{~nm})$. Similar results were also found by treating the gold particle array as an effective medium layer and using much faster 1D-TM calculations. On the contrary, 3D-TM calculations were needed to reveal the onset of reflectance in backward diffraction direction, for periods higher than $\sim 180 \mathrm{~nm}$, in agreement with the predicted value. At the angles of leaky waveguide resonances, as the period was increased from $\sim 180 \mathrm{~nm}$, the backscattered reflectance increased sharply up to a maximum of $\sim 1.5 \times 10^{-4}$, then decreased and eventually vanished for periods higher than $\sim 215 \mathrm{~nm}$. We also checked that, in our simulations, there was no light radiated in all other diffraction directions, except for the backward one. Experimentally, the measured backscattered power was found to be sensitive to vapour substances, with clear ability to discriminate between hydrating and dehydrating vapours (Fig. 3). This sensitivity was confirmed by simulations too. Simulations predicted that the optimal particle lateral separation was $a=185 \sim 190 \mathrm{~nm}$ : this range corresponded to gold filling factors $~ 10$ times lower than in experiments. Therefore, in the random particle arrangements of the actual samples, only those particles that were separated by such distances are thought to contribute to the observed effects. Further calculations on random nanoparticle arrangements are in progress to confirm this prediction.
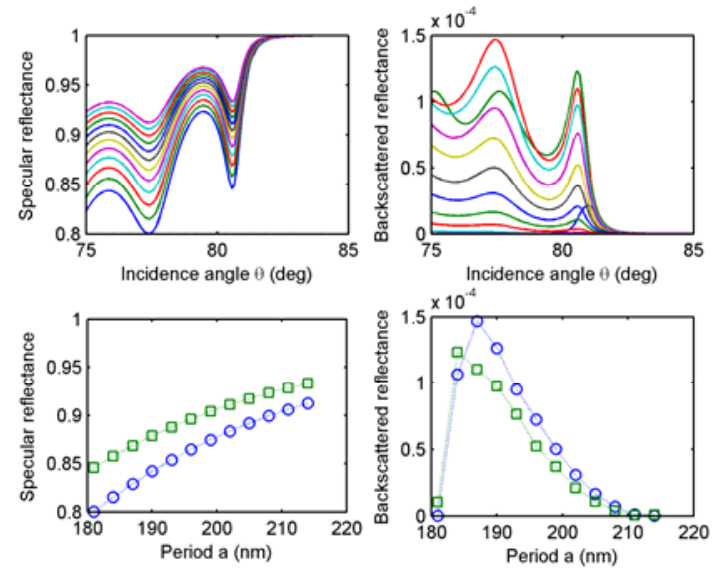

Fig. 2 Specular (top left) and backscattered (top right) reflectance angular spectra for various periods of gold nanoparticle array; specular (bottom left) and backscattered (bottom right) reflectance at first (circles) and second (squares) resonant angles ( $\theta_{1}=77.4 \mathrm{deg}$ and $\theta_{1}=80.5 \mathrm{deg}$, respectively) as functions of the period of gold nanoparticle array.

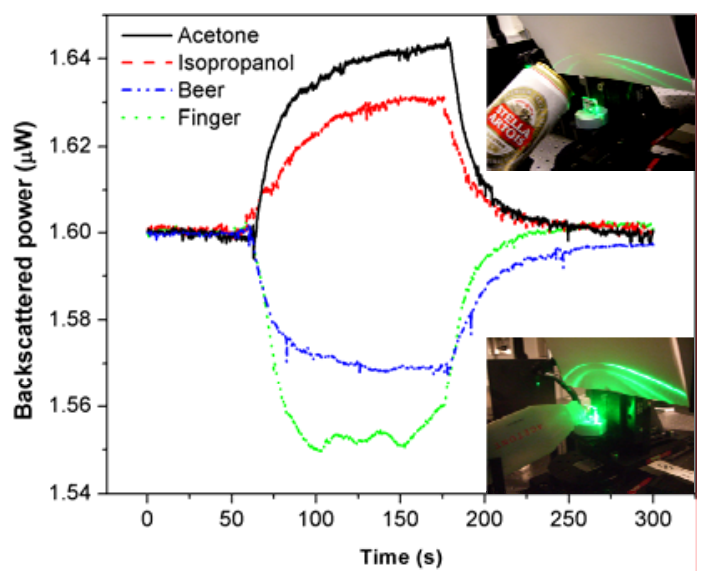

Fig. 3 Evolution of bacsckattered power from a poled nanocomposite when vapour from bottle (see insets) or finger is first approached and then kept away from the sample. Note the increase or decrease of signal with hydrating (beer, finger) or dehydrating (acetone, isopropanol) substances, respectively.

\section{Conclusions}

We showed theoretically that thermally poled plasmonic nanocomposites exhibited enhanced backscattering at grazing incidence and we presented an application of this concept for selective vapour sensing.

\section{References}

1. $\quad$ Corbari C., Beresna M., Deparis O. and Kazansky P. G., "Leaky-modes excitation in thermally poled nanocomposite glass and their exploitation for saturable absorption", Bragg Gratings, Photosensitivity and Poling in Glass Waveguides (BGPP) 2010 , paper BTuB7.

2. Beresna M., Deparis O., Carvalho I. C. S., Takahashi S., Zayats A. V. and Kazansky P. G., "Poling-assisted fabrication of plasmonic nanocomposite devices in glass," Advanced Materials, 2010. (accepted for publication). 